\title{
Insulin Resistance, the Metabolic Syndrome and Type 2 Diabetes
}

\author{
Claire Levy-Marchal \\ INSERM Unit 690, Robert Debré Hospital, Paris, France \\ clairelm@rdebre.inserm.fr
}

\begin{abstract}
Experimental studies on the mechanisms of insulin resistance are exciting this year. Just look at the physiology of PPAR- $\delta$ and the newly described functions of insulin and also vaspin coming on the market! There is not a single month without a new hormone or a new function of the adipose tissue being brought to light. Progress made over the past few months on the understanding of insulin resistance itself and on the pathology and the connections between fat, muscle, liver and $\beta$-cell are just incredible. Mirroring these breakthroughs and supported by the increasing and alarming extension of obesity, numerous clinical studies are related to risk factors for insulin resistance and the metabolic syndrome in childhood. The selection of publications is sometimes critical and here we try to focus on specific and pediatric aspects of the debate 'whether the metabolic syndrome is for real', pointing to important subjects relevant to clinical practice.
\end{abstract}

\section{Mechanism of the year}

\section{Antagonistic actions of ecdysone and insulins determine final size in Drosophila}

Colombani J, Bianchini L, Layalle S, Pondeville E, Dauphin-Villemant C, Antoniewski C, Carre C, Noselli S, Leopold P CNRS/University of Nice-Sophia Antipolis, UMR6543, Nice, France

Science 2005:310:667-670

Background: All animals coordinate growth and maturation to reach their final size and shape. In insects, insulin family molecules control growth and metabolism, whereas pulses of the steroid 20-hydroxyecdysone initiate major developmental transitions.

Methods: Construction of a genetic model for modulation of the concentrations of ecdysone.

Results: The authors show that 20-hydroxyecdysone signaling also negatively controls animal growth rates by impeding general insulin signaling involving localization of the transcription factor dFOXO and transcription of the translation inhibitor 4E-BP. The authors also demonstrate that the larval fat body, equivalent to the vertebrate liver, is a key relay element for ecdysone-dependent growth inhibition.

Conclusion: Ecdysone counteracts the growth-promoting action of insulin, thus forming a humoral regulatory loop that determines organismal size.

This experimental work dissects the mechanisms of growth of the larva of the Drosophila with opposite actions of steroids and insulin/IGFs. The observations reveal a key role of the fat body of the larva, integrating nutritional inputs and controlling the insulin-signaling pathway to modulate organismal growth. This is an additional and fascinating example of interferences of nutrition, metabolism (and insulin) and growth. How, then, is growth connected to developmental timing? These findings place this hormone in a central position for coordinating these two key processes and controlling organismal size. 


\title{
Characterization of insulin secretion and resistance in type 2 diabetes of adolescents
}

\author{
Druet C, Tubiana-Rufi N, Chevenne D, Rigal O, Polak M, Levy-Marchal C \\ Institut National de la Sante et de la Recherche Médicale, Unité 690, Hôpital Robert-Debré, Paris, France \\ drucel@yahoo.fr \\ J Clin Endocrinol Metab 2006;91:401-404
}

Background: Type 2 diabetes (T2D) in obese children is an emerging problem, including in Europe. Its presentation at diagnosis very often differs from that in adults.

Aim: The objective of this study was to investigate the relative contributions of the two components of $\mathrm{T} 2 \mathrm{D}$, insulin resistance and insulin secretion, early in the history of the disease in adolescents.

Methods: Six obese adolescents with T2D were included 2 months to 4.3 years after diagnosis $(5$ girls and 1 boy; median age 15.4 years; median body mass index 4.4 SD). Peripheral and hepatic insulin sensitivity was evaluated with euglycemic hyperinsulinemic $\left(40 \mathrm{mU} / \mathrm{m}^{2} \cdot \mathrm{min}\right)$ clamp. First-phase insulin release was evaluated after intravenous glucose stimulation. A graded intravenous glucose infusion and an arginine test were performed to measure insulin secretion.

Results: All patients showed decreased peripheral glucose uptake to the same extent. Five patients showed hepatic insulin resistance. First-phase insulin release was very low in 2 patients. Three patients showed an exaggerated insulin response under graded glucose infusion and preserved secretion under arginine stimulation. Three other patients, with elevated fasting plasma glucose levels, demonstrated a very low insulin response under glucose stimulation and a low insulin response under arginine stimulation.

Conclusion: These data emphasize that together with marked insulin resistance, the failure of $\beta$-cell function is a major component in the course of T2D in childhood.

\section{Decreased $\beta$-cell function in overweight Latino children with impaired fasting glucose}

Weigensberg MJ, Ball GD, Shaibi GQ, Cruz ML, Goran MI

Department of Pediatrics, Keck School of Medicine, University of Southern California, Los Angeles, Calif., USA

Diabetes Care 2005;28:2519-2524

Background: To determine whether overweight Latino children with impaired fasting glucose $(\geq 100 \mathrm{mg} / \mathrm{dl}$ ) have increased insulin resistance or decreased $\beta$-cell function compared with those with normal fasting glucose.

Methods: 207 healthy overweight Latino children, aged 8-13 years, with a family history of type 2 diabetes were studied. Fasting and 2-hour glucose and insulin were assessed by oral glucose tolerance test. Insulin sensitivity $\left(\mathrm{S}_{\mathrm{i}}\right)$, the acute insulin response to glucose (AIRg), and the disposition index (DI; an index of $\beta$-cell function) were determined using the insulin-modified intravenous glucose tolerance test and minimal modeling. Body composition was determined by dual-energy X-ray absorptiometry.

Results: There were no differences in body composition between normal fasting glucose $(\mathrm{n}=182)$ and IFG $(n=25)$ children. Compared with children with normal fasting glucose, children with impaired fasting glucose had higher fasting and 2-hour glucose values and higher fasting insulin. After adjusting for covariates, children with impaired fasting glucose had no difference in $S_{i}$ but $15 \%$ lower DI than normal fasting glucose children $(2,224 \pm 210$ vs. $2,613 \pm 76, \mathrm{p}<0.05)$. Multivariate linear regression showed that AIRg and DI, but not $\mathrm{S}_{\mathrm{i}}$, were significant predictors of fasting blood glucose.

Conclusion: In overweight Latino adolescents with a family history of type 2 diabetes, impaired fasting glucose is associated with impaired $\beta$-cell function and therefore may identify children likely to be at risk for progression to type 2 diabetes. The actual risk of progression of impaired fasting glucose to type 2 diabetes remains to be determined by prospective longitudinal studies.

These two papers emphasize the role of impaired insulin secretion in the deterioration of glucose tolerance. Using two different approaches in two study populations, the data point to decreased insulin secretion very early in the course of glucose tolerance deterioration, especially in the case of family history of type 2 diabetes in obese children. These observations are important not only to describe 
the natural course of the disease in children but also from a therapeutic point of view. There are currently no guidelines on the treatment of type 2 diabetes in children and results of therapeutic trials are scanty. Beside insulin sensitizers there will certainly be room for insulin therapy in children with type 2 diabetes. Pre-diabetes, IFG and IGT may identify children likely to be at similar degrees of risk for progression to type 2 diabetes. The actual risk of progression to type 2 diabetes and the predictive value of impaired insulin secretion in such children remain to be determined by prospective longitudinal studies.

\section{Clinical practice}

\section{Ghrelin levels before and after reduction of overweight due to a low-fat high-carbohydrate diet in obese children and adolescents}

Reinehr T, Roth CL, Alexy U, Kersting M, Kiess W, Andler W

Int J Obes (Lond) 2005;29:362-368

Background: There are conflicting results for ghrelin changes in reduction of overweight. Increasing ghrelin levels in weight reduction are considered to be responsible for compensatory mechanisms that make the reduction of overweight unlikely to be sustained.

Methods: The authors have analyzed fasting serum ghrelin levels, weighed dietary record and, as biochemical markers of clinically relevant reduction of overweight, leptin, adiponectin and insulin levels and insulin resistance measured by homeostasis model assessment (HOMA) at baseline and after a 1year outpatient weight reduction program based on a high-carbohydrate and low-fat diet in 37 obese children (median age 10 years). These children were divided into two subgroups according to their degree of weight loss (substantial reduction of overweight: decrease in SDS-BMI $>$ or $=0.5$ ). Furthermore, ghrelin levels were analyzed in 16 normal-weight children.

Results: Obese children demonstrated significantly $(\mathrm{p}<0.001)$ lower ghrelin levels compared to normalweight children. Daily caloric intake $(\mathrm{p}=0.004)$ and percentage fat content decreased significantly $(\mathrm{p}<0.001)$, while percentage carbohydrate content increased significantly $(\mathrm{p}=0.003)$ between baseline and 1-year follow-up in the obese children. The substantial reduction of overweight in 16 children (median SDS-BMI $=-0.7$ ) was associated with significant changes in insulin resistance (median decrease of HOMA $27 \% ; \mathrm{p}=0.013$ ), insulin (median decrease $25 \%, \mathrm{p}=0.036$ ), adiponectin (median increase $15 \% ; \mathrm{p}=0.003$ ), and leptin levels (median decrease $19 \% ; \mathrm{p}=0.023$ ), while there were no significant changes in ghrelin levels (median increase $4 \% ; p=0.326$ ). In the 21 children without substantial reduction of overweight (median SDS-BMI $=-0.3$ ), there were no significant changes in insulin resistance and in insulin, adiponectin, leptin and ghrelin levels.

Conclusion: In obese children, low-fat high-carbohydrate diet-induced weight loss does not change ghrelin secretion, but significantly decreases leptin levels, increases adiponectin levels and improves insulin resistance determined by significantly decreased insulin resistance indices as well as lowered serum insulin levels.

\section{Resistin concentrations before and after weight loss in obese children}

Reinehr T, Roth CL, Menke T, Andler W

Vestische Hospital for Children and Adolescents Datteln, University of Witten/Herdecke, Datteln, Germany

Int J Obes (Lond) 2006;30:297

Background: The physiology of resistin has not yet been extensively studied, in particular in childhood.

Methods: Age, pubertal stage, gender, weight status (standard deviation score-body mass index (SDSBMI)), resistin levels, and insulin resistance index calculated by homeostasis model assessment (HOMA) were evaluated in 63 obese children and compared to 36 lean children. Furthermore, the changes of weight status (SDS-BMI, percentage body fat based on skinfold measurements), waist-to-hip ratio, resistin, and HOMA over a 1-year period were analyzed in 38 obese children.

Results: The resistin levels did not significantly $(\mathrm{p}=0.079)$ differ between obese (median resistin $8.7 \mathrm{ng} / \mathrm{ml}$ ) and lean children (median resistin $9.7 \mathrm{ng} / \mathrm{ml}$ ). Resistin concentrations were independent of 
age and pubertal stage, but girls demonstrated significantly higher resistin levels than boys $(\mathrm{p}=0.021)$. There were no significant correlations between changes of resistin and changes of SDS-BMI $(r=0.14$, $\mathrm{p}=0.198)$, changes of percentage body fat $(\mathrm{r}=-0.01, \mathrm{p}=0.466)$, changes of waist-to-hip ratio $(\mathrm{r}=0.17, \mathrm{p}=0.141)$, and changes of insulin resistance index $(\mathrm{r}=0.01, \mathrm{p}=0.472)$ over the 1-year period.

Conclusions: Girls demonstrated higher resistin concentrations than boys. The data do not support a relationship between resistin, insulin resistance index, and weight status in childhood.

These papers highlight the physiological roles in humans of two recently described hormones: ghrelin and resistin. The physiological situation is the changes induced by insulin resistance in obese children after significant weight loss. Following the improvement in insulin sensitivity, circulating levels of adiponectin and leptin were consequently affected. But the levels of both ghrelin and resistin were not. Moreover, circulating levels of resistin were not different between lean and obese children and were not affected by major determinants of obesity. Ghrelin is mostly secreted by the stomach and implicated in satiety. Its implication in insulin sensitivity is probably of minor relevance in metabolic complications of obesity in humans. Data are more surprising for resistin, which was found not correlated to age, weight or insulin resistance status, highlighting that we need to know much more about this strange hormone. The role of resistin in rodent physiology remains less ambiguous than its role in humans.

\section{Insulin resistance and metabolic syndrome: definitions and epidemiological data}

\section{Ethnic differences in insulin resistance and body composition in United Kingdom adolescents}

Ehtisham S, Crabtree N, Clark P, Shaw N, Barrett T

Department of Diabetes and Endocrinology, Birmingham Children's Hospital, Birmingham, UK

s.ehtisham@bham.ac.uk

J Clin Endocrinol Metab 2005;90:3963-3969

Background: Type 2 diabetes is increasingly recognized in childhood, occurring more frequently in the United Kingdom in South Asians and in girls. South Asian children have been shown to be more insulin-resistant than white European children, and girls more insulin-resistant than boys. It is not clear how these sex and ethnic differences relate to body composition in childhood.

Aim: The goal was to evaluate sex and ethnic differences in insulin sensitivity and body composition in healthy adolescents.

Methods: Cross-sectional cohort study. 129 healthy white European and South Asian 14- to 17-year-old adolescents participated. Body composition was assessed by anthropometry and dual-energy x-ray absorptiometry, and insulin sensitivity by homeostasis model assessment.

Results: South Asian adolescents were less insulin-sensitive than white European adolescents (52.4 vs. $58.9 \%, \mathrm{p}<0.05)$, with a trend toward lower insulin sensitivity in girls. South Asian adolescents had significantly more body fat than white European adolescents (girls, 30.6 vs. $26.0 \%$, p $<0.005$; boys, 20.8 vs. $14.8 \%, \mathrm{p}<0.001$ ), with more central fat (waist-thigh ratio in girls, 1.36 vs. $1.25, \mathrm{p}<0.001$; boys, 1.52 vs. $1.42, \mathrm{p}<0.001)$. The sex-ethnic differences in insulin sensitivity were no longer seen when body fat was included as a covariate.

Conclusion: Ethnic differences in insulin sensitivity are associated with ethnic differences in body fat. South Asian adolescents are more insulin-resistant, with more body fat than white European adolescents, which may contribute to their increased risk of developing type 2 diabetes. 


\title{
Comparison of metabolic syndrome prevalence using six different definitions in overweight pre-pubertal children enrolled in a weight management study
}

\author{
Golley RK, Magarey AM, Steinbeck KS, Baur LA, Daniels LA \\ Department of Nutrition and Dietetics, Flinders University, Adelaide, Australia \\ Int J Obes (Lond) 2006; doi: 10.1038/sj.ijo.0803195
}

Aim: To assess the implications of variation in metabolic syndrome (MS) definition (biochemical and anthropometric indicators) on MS prevalence estimates in a population of overweight and mildly obese children.

Methods: Cross-sectional study. 99 (64 girls) overweight or mildly obese, but otherwise healthy, prepubertal 6- to 9-year-olds were recruited for a randomized controlled trial of weight management. Height, weight and waist circumference were measured with BMI and waist z-scores calculated. Fasting cholesterol and fractions, glucose and insulin were measured, together with systolic and diastolic blood pressure (BP). Anthropometric and metabolic indicators were classified as normal or elevated using adult- or child-specific cut points with clustering of MS indicators also assessed using two adult and three child-specific definitions.

Results: A total of $0-4 \%$ of subjects were classified with MS when adult definitions were applied. This increased to between 39 and $60 \%$ using child-specific definitions, varying according to whether hyperinsulinemia was central to the MS classification. Systolic BP, triglycerides, total cholesterol, high-density lipoprotein cholesterol and waist $z$-score increased across insulin quartiles $(p<0.05)$. The use of body mass index and waist circumference in the MS definition classified the same subjects.

Conclusions: The classification of MS in children depends strongly on the definition chosen, with MS prevalence estimates higher if insulin is part of the definition and child-specific cut points for metabolic indicators are used. Hyperinsulinemia and MS are common consequences of childhood obesity but they are not commonly part of the assessment or management plan for weight management in children. There is a need for the establishment of normal insulin ranges and consistent definition of MS in childhood and adolescence.

\section{Insulin resistance and impaired glucose tolerance in obese children and adolescents referred to a tertiary-care center in Israel}

Shalitin S, Abrahami M, Lilos P, Phillip M

Institute for Endocrinology and Diabetes, Schneider Children's Medical Center of Israel, Petah Tiqva, Israel shalitin@netvision.net.il

Int J Obes (Lond) 2005:29:571-578

Aim: To establish the prevalence of insulin resistance and impaired glucose tolerance (IGT) and their determinants in a cohort of obese children and adolescents.

Methods: A retrospective design was used. The study group included 234 patients with a body mass index (BMI) greater than the 95th percentile for age and gender and 22 patients with a BMI between the 85 th and 95th percentile for age and gender referred for evaluation to a major tertiary-care center in Israel. Ages ranged from 5 to 22 years. Estimates of insulin resistance (homeostatic model assessment (HOMA-IR)); insulin sensitivity (ratio of fasting glucose (GF) to fasting insulin (IF) (GF/IF), the quantitative insulin sensitivity check index (QUICKI)), and pancreatic $\beta$-cell function (HOMA-derived $\beta$-cell function (HOMA \%B)) were derived from fasting measurements. An oral glucose tolerance test (OGTT) was performed in 192 patients to determine the presence of IGT.

Results: Insulin resistance was detected in $81.2 \%$ of the patients, IGT in $13.5 \%$, and silent diabetes in 1 adolescent girl. Only 2 patients with IGT also had impaired fasting glucose (IFG). The prevalence of IGT was higher in adolescents than prepubertal children (14.7 vs. 8.6\%). GF/IF and QUICKI decreased significantly during puberty $(\mathrm{p}<0.005)$, whereas HOMA-IR and HOMA \%B did not. Insulin resistance and insulin sensitivity indexes were not associated with ethnicity, presence of acanthosis nigricans or family history of type 2 diabetes. Patients with obesity complications had lower insulin sensitivity indexes than those without $(\mathrm{p}=0.05)$. Compared with subjects with normal glucose tolerance (NGT), patients with IGT had a higher degree of insulin resistance and worse metabolic profile. 
Conclusion: Insulin resistance is highly prevalent in obese children and adolescents. The onset of IGT is associated with the development of severe insulin resistance; an OGTT is required in all subjects at high risk. Longitudinal studies are needed to identify the metabolic precursors and the natural history of the development of type 2 diabetes in these patients.

This year's clinical papers on the metabolic complications of obesity emphasize the difficulties of evaluating the syndrome in children. There are several reasons for this:

- The lack of standardized definition [1]. It is not only the lack of consensual definition of the metabolic syndrome but also the difficulties to select cut-off values in children. The reference values should vary with country or ethnicity and we have no longitudinal data in children to establish cut-off values based on prediction of cardiovascular risk. Little is known with respect of tracking of adiposity and metabolic consequences from very young ages to adulthood.

- Whatever the definitions, all reports indicate that insulin resistance and the metabolic complications are driven by obesity and the degree of adiposity even among obese children [2-4]. The deterioration of glucose tolerance is accompanied by a severe degree of insulin resistance $[5,6]$.

- The ethnic differences seen in adulthood have their origins in childhood. The ethnic differences in both insulin sensitivity and body composition seen in healthy adolescents may account for the observed sex and ethnic differences in type 2 diabetes prevalence in childhood.

One of the questions that has been vigorously debated in the literature this year is whether we need a definition of the metabolic syndrome? [7-11]. For some authors, the cardiovascular risk conferred by the metabolic risk is not different or more precise than that conferred by the summation of the risks associated with each individual component (glucose intolerance, lipids profile, elevated blood pressure, obesity). On the other hand, the concept of the metabolic syndrome brings the physicians' attention to treat the patient as a whole rather than each individual component. The question of the causal relationship between cardiovascular diseases and the metabolic syndrome vs. insulin resistance is not solved yet, and might get a similar answer as the egg/hen debate.

Fetal origins of adult diseases

\section{Glucose tolerance at age 58 and the decline of glucose tolerance in comparison with age 50 in people prenatally exposed to the Dutch famine}

de Rooij SR, Painter RC, Roseboom TJ, Phillips DI, Osmond C, Barker DJ, Tanck MW, Michels RP,

Bossuyt PM, Bleker OP

Department of Clinical Epidemiology and Biostatistics, Academic Medical Center, University of Amsterdam,

Amsterdam, The Netherlands

s.r.derooij@amc.uva.nl

Diabetologia 2006;49:637-643

Background: People who were small at birth have an increased risk of type 2 diabetes in later life. People who were in utero during the Dutch famine had decreased glucose tolerance and raised insulin concentrations at age 50 .

Aim: To evaluate whether prenatal famine exposure leads to more rapid progression of impaired glucose/insulin homeostasis with increasing age.

Methods: An OGTT was performed in 702 men and women at age 50 and in 699 men and women at age 58, all born as term singletons immediately before, during or after the 1944-1945 Dutch famine.

Results: People who had been exposed to famine in utero had significantly higher 120-min glucose concentrations at age 58 compared with people who had not been exposed to famine (difference $=$ $0.4 \mathrm{mmol} / \mathrm{l}, 95 \%$ CI $0.1-0.7$, adjusted for sex and BMI). Glucose tolerance deteriorated between the age of 50 and 58 . The unadjusted 120 -min glucose concentrations rose by $0.2 \mathrm{mmol} / \mathrm{l}(95 \% \mathrm{CI} 0.0-0.4)$, while 120-min insulin concentrations had increased by $64 \mathrm{pmol} / \mathrm{l}$ (95\% CI 48-82). There were no differences in the rates of glucose and insulin level increase between the famine-exposed group and the unexposed group $(\mathrm{p}=0.28$ for the difference in increase in glucose concentrations and $\mathrm{p}=0.09$ for insulin concentrations). 
Conclusion: Although it is confirmed that undernutrition during gestation is linked to decreased glucose tolerance, the effect does not seem to become more pronounced at age 58 as compared with age 50 .

This paper confirms the association between the Dutch famine [12], low birth weight and the later deterioration of glucose tolerance, and it is the first to show the degree of transition from NGT to IGT in subjects exposed to the famine. So the risk of disorders of glucose tolerance is higher but the rate of progression does not seem different from the non-exposed ones. This points to programming being an early event in life as indicated in the next paper.

\section{Longitudinal changes in insulin sensitivity and secretion from birth to age 3 years in small- and appropriate-for-gestational-age children}

Mericq V, Ong KK, Bazaes R, Pena V, Avila A, Salazar T, Soto N, Iniguez G, Dunger DB

Institute of Maternal and Child Research, University of Chile, Santiago, Chile

vmericq@med.uchile.cl

Diabetologia 2005;48:2609-2614

Background: Insulin resistance and type 2 diabetes risk in human subjects who were small-for-gestationalage (SGA) at birth may be a consequence of rapid early postnatal weight gain.

Methods: The authors prospectively studied early changes in fasting insulin sensitivity and insulin secretion, assessed by a short intravenous glucose tolerance test that was conducted several times from birth to 3 years of age in 55 SGA (birth weight below 5th percentile) newborns and in 13 newborns with a birth weight appropriate for gestational age (AGA).

Results: Most SGA infants showed postnatal upward weight centile crossing and by 3 years were similar in size to AGA infants. SGA infants had lower pre-feed insulin levels at postnatal age $48 \mathrm{~h}$ than AGA infants (median 34.4 vs. $59.7 \mathrm{pmol} / \mathrm{l}, \mathrm{p}<0.05$ ), but by the age of 3 years they had higher fasting insulin levels (median 38.9 vs. $23.8 \mathrm{pmol} / \mathrm{l}, \mathrm{p}<0.005$ ), which were related to rate of weight gain between 0 and 3 years $(\mathrm{r}=0.47, \mathrm{p}=0.0003)$. First-phase insulin secretion did not differ between SGA and AGA infants, but SGA infants had a lower glucose disposition index ( $\beta$-cell compensation) (median 235 vs. $\left.501 \mathrm{~min} \mathrm{mmol}^{-1} \mathrm{1}^{-1}, \mathrm{p}=0.02\right)$, which persisted after allowing for postnatal weight gain $(\mathrm{p}=0.009)$. Conclusion: SGA infants showed a marked transition from lower pre-feed insulin and increased insulin sensitivity at birth to insulin resistance over the first 3 years of life. This transition was related to rapid postnatal weight gain, which could indicate a propensity to central fat deposition. The additional observation of reduced compensatory $\beta$-cell secretion underlines the need for long-term surveillance of glucose homeostasis in all SGA subjects, whether or not they show postnatal catch-up growth.

This paper confirms a previous observation made by the same group that insulin resistance associated with reduced fetal growth is visible very early in life [13]. It is only 15 years ago that this puzzling observation was reported in the literature and there is now accumulating evidence that programming has something to do with adiposity as soon as the period of catch-up growth starts in the very first years of life. The issue of whether insulin secretion is affected by fetal growth is more debated in these children. Indeed, first-phase insulin release is highly variable and remains the most common way of evaluating insulin secretion in clinical situations. The prospective follow-up of these children will certainly yield valuable information.

\section{Gestational glucose tolerance and risk of type 2 diabetes in young Pima Indian offspring}

Franks PW, Looker HC, Kobes S, Touger L, Tataranni PA, Hanson RL, Knowler WC

Genetics Group, MRC Epidemiology Unit, University of Cambridge, Cambridge, UK

paul.franks@mrc-epid.cam.ac.uk

Diabetes 2006;55:460-465

Background: The in utero environment is a powerful risk factor for type 2 diabetes in offspring, but little is known about the risk conveyed by non-diabetic gestational glucose levels.

Methods: This issue was explored in 911 non-diabetic Pima Indian mothers and 1,436 of their children. Associations were assessed in multivariate models between maternal third trimester glucose tolerance 
and indexes of body composition and glycemic control in their children. At parturition, the mothers' ages ranged from 14 to 43 years. Offspring were studied at age $0-39$ years.

Results: An SD (1.3 mmol/l) of maternal glucose was associated with $56 \mathrm{~g}$ higher birth weight $(\mathrm{p}=0.0002)$. This effect persisted when only offspring of normal glucose tolerant mothers were examined $(57 \mathrm{~g}$, $\mathrm{p}<0.0001)$. In Cox proportional hazards models, the adjusted hazard rate ratio for offspring risk of diabetes per SD maternal glucose was 1.6 (95\% CI 1.3-2.0, p $<0.0001)$. When only offspring of normal glucose-tolerant mothers were examined, the risk was reduced but remained significant (1.3, $\mathrm{p}=0.026)$.

Conclusion: Increasing maternal glycemia during pregnancy is associated with increased risk of diabetes in Pima Indian offspring, even when mothers are normal glucose tolerant during pregnancy.

The relationship between gestational diabetes and later risk of type 2 diabetes in the offspring is well known. This paper impressively shows that the relationship between the glycemic level during the third trimester of pregnancy and the risk of type 2 diabetes in the offspring extends to the normal range of glucose levels. It looks as if any increase in blood glucose increases the risk and decreases the age of onset of diabetes in the offspring. Because of the very high incidence rates of type 2 diabetes in this population and because of an early onset, the Phoenix group was actually the first to demonstrate the role of the fetal environment in the decreasing age of onset of diabetes in the Pima Indians [14, 15]. Prevention of offspring type 2 diabetes may require strategies that focus on improving gestational glucose tolerance even within the normal range.

\section{New mechanisms}

\section{PPAR- $\delta$ regulates glucose metabolism and insulin sensitivity}

Lee C-H, Olson P, Hevener A, Mehl I, Chong L-W, Olefsky JM, Gonzalez FJ, Ham J, Kang H, Peters JM, Evans RM

Department of Genetics and Complex Diseases, Harvard School of Public Health, Boston, Mass., USA

Proc Natl Acad Sci USA 2006;103:3444-3449

Background: The metabolic syndrome is a collection of obesity-related disorders. The peroxisome proliferatoractivated receptors (PPARs) regulate transcription in response to fatty acids and, as such, are potential therapeutic targets for these diseases.

Results: PPAR- $\delta$ (NR1C2) knockout mice are metabolically less active and glucose-intolerant, whereas receptor activation in $\mathrm{db} / \mathrm{db}$ mice improves insulin sensitivity. Euglycemic-hyperinsulinemic-clamp experiments further demonstrate that a PPAR- $\delta$-specific agonist suppresses hepatic glucose output, increases glucose disposal, and inhibits free fatty acid release from adipocytes. Unexpectedly, gene array and functional analyses suggest that PPAR- $\delta$ ameliorates hyperglycemia by increasing glucose flux through the pentose phosphate pathway and enhancing fatty acid synthesis. Coupling increased hepatic carbohydrate catabolism with its ability to promote lipid oxidation in muscle allows PPAR- $\delta$ to regulate metabolic homeostasis and enhance insulin action by complementary effects in distinct tissues.

Conclusion: The combined hepatic and peripheral actions of PPAR- $\delta$ suggest new therapeutic approaches to treat type 2 diabetes.

The glucose-fatty acid cycle proposed by Randle et al. [16] postulates that increased fatty acid oxidation results in decreased carbohydrate utilization. PPAR- $\delta$, a fatty acid receptor able to induce lipid oxidation, enhances glucose utilization by its ability to promote glucose flux through the pentose phosphate shunt and to stimulate hepatic fatty acid synthesis. This paper contributes to unravel the network of the PPARs. All PPARs are lipid-activated transcription factors and are controlling substrate utilization through regulating distinct transcriptional programs in different tissues. PPAR- $\alpha$ in the liver stimulates fatty acid oxidation and indirectly promotes neoglucogenesis. PPAR- $\gamma$ promotes synthesis and storage of lipids in the adipose tissue. PPAR- $\delta$ induces lipid oxidation in the muscle and glycolysis and lipogenesis in the liver. This study implicates PPAR- $\delta$ as a bona fide target to treat insulin resistance and as a candidate for population-based screening for genetic polymorphisms that predispose to this disease. 


\title{
Activation of the nuclear receptor FXR improves hyperglycemia and hyperlipidemia in diabetic mice
}

\author{
Zhang Y, Lee FY, Barrera G, Lee H, Vales C, Gonzalez FJ, Willson TM, Edwards PA \\ Department of Biological Chemistry, University of California, Los Angeles, Calif., USA \\ Proc Natl Acad Sci 2006; 103:1006-1011
}

Background: Farnesoid X receptor (FXR) plays an important role in maintaining bile acid and cholesterol homeostasis. Here it is demonstrated that FXR also regulates glucose metabolism.

Results: Activation of FXR by the synthetic agonist GW4064 or hepatic overexpression of constitutively active FXR by adenovirus-mediated gene transfer significantly lowered blood glucose levels in different mice strains. Consistent with these data, FXR null mice exhibited glucose intolerance and insulin insensitivity. Activation of FXR in $\mathrm{db} / \mathrm{db}$ mice repressed hepatic gluconeogenic genes and increased hepatic glycogen synthesis and glycogen content by a mechanism that involves enhanced insulin sensitivity. Conclusion: In view of its central roles in coordinating regulation of both glucose and lipid metabolism, the authors propose that FXR agonists are promising therapeutic agents for treatment of diabetes mellitus.

\begin{abstract}
Numerous studies have suggested that hyperlipidemia is closely linked to cardiovascular disease. In addition, diabetes is associated with a 2- to 4-fold increase in coronary heart disease. FXR has known metabolic function in cholesterol and triglyceride metabolism, but its role in carbohydrate metabolism is brand new. The pronounced hypoglycemic effect is due to the activation of the hepatic FXR, which in turn increases insulin sensitivity. In view of its hypoglycemic and hypolipidemic properties, this newly described effect opens a wide potential for treatment of diabetes and prevention of complications that include coronary heart disease.
\end{abstract}

\section{New gene}

\section{Variant of transcription factor 7-like 2 (TCF7L2) gene confers risk of type 2 diabetes}

Grant SFA ${ }^{a}$, Thorleifsson $\mathrm{G}^{\mathrm{a}}$, Reynisdottir $\mathrm{I}^{\mathrm{a}}$, Benediktsson $\mathrm{R}^{\mathrm{b}, \mathrm{c}}$, Manolescu $\mathrm{A}^{\mathrm{a}}$, Sainz Ja, Helgason $\mathrm{A}^{\mathrm{a}}$, Stefansson $\mathrm{H}^{\mathrm{a}}$, Emilsson Va, Helgadottir $A^{a}$, Styrkarsdottir Ua, Magnusson KPa, Walters GBa, Palsdottir Ea, Jonsdottir Ta, Gudmundsdottir Ta, Gylfason $A^{a}$, Saemundsdottir Ja, Wilensky RL' ${ }^{d}$, Muredach Pd, Reilly MPd, Rader DJd, Bagger $Y^{e}$, Christiansen $C^{e}$, Gudnason $V^{b}$, Sigurdsson $G^{b, c}$, Thorsteinsdottir $U^{a}$, Gulcher JR ${ }^{a}$, Kong A ${ }^{a}$, Stefansson $K^{a}$ adeCODE genetics, Reykjavik; 'bcelandic Heart Association, Kopavogur, and 'National University Hospital, Reykjavik, Iceland; dUniversity of Pennsylvania School of Medicine, Philadelphia, Pa., USA, and 'Center for Clinical and Basic Research A/S, Ballerup, Denmark

Nat Genet 2006;38:320-323

Background: A suggestive linkage of type 2 diabetes mellitus to chromosome $10 \mathrm{q} 1$ was previously reported by the same group.

Methods: 228 microsatellite markers in Icelandic individuals with type 2 diabetes and controls throughout a $10.5-\mathrm{Mb}$ interval on $10 \mathrm{q}$ were genotyped.

Results: A microsatellite, DG10S478, within intron 3 of the transcription factor 7-like 2 gene (TCF7L2; formerly TCF4) was associated with type 2 diabetes $\left(\mathrm{p}=2.1 \times 10^{-9}\right)$. This was replicated in a Danish cohort $\left(\mathrm{p}=4.8 \times 10^{-3}\right)$ and in a US cohort $\left(\mathrm{p}=3.3 \times 10^{-9}\right)$. Compared with non-carriers, heterozygous and homozygous carriers of the at-risk alleles (38 and $7 \%$ of the population, respectively) have relative risks of 1.45 and 2.41 .

Conclusion: The effect detected corresponds to a population-attributable risk of $21 \%$. The TCF7L2 gene product is a high mobility group box-containing transcription factor previously implicated in blood glucose homeostasis. It is thought to act through regulation of proglucagon gene expression in enteroendocrine cells via the Wnt-signaling pathway. 
This paper is interesting for at least three reasons:

- The innovative genetic technology.

- The high relative risk conferred by the mutated allele is of relevant magnitude and of clinical interest; moreover, the risk has been replicated in three different and independent populations conferring some robustness to the observation.

- The underlying pathway refers to GLP-1 and the entero-endocrine axis.

It is unclear why this polymorphism remained unidentified. One reason is that it did not appear as a first-choice candidate gene. However, the magnitude of the risk is equal to the one conferred by the Pro12Ala polymorphism of the PPAR- $\gamma 2[17,18]$, which had remained unequalled for type 2 diabetes.

\title{
New hormone
}

\section{Visceral adipose tissue-derived serine protease inhibitor: a unique insulin-sensitizing adipocytokine in obesity}

\author{
Hida K, Wada J, Eguchi J, Zhang H, Baba M, Seida A, Hashimoto I, Okada T, Yasuhara A, Nakatsuka A, Shikata K, \\ Hourai S, Futami J, Watanabe E, Matsuki Y, Hiramatsu R, Akagi S, Makino H, Kanwar YS \\ Department of Medicine and Clinical Science, Okayama University Graduate School of Medicine and Dentistry, \\ Okayama, Japan
}

Proc Natl Acad Sci USA 2005;102:10610-10615

Background: There is a rapid global rise in obesity, and the link between obesity and diabetes remains somewhat obscure. An adipocytokine designated as visceral adipose tissue-derived serpin (vaspin) was identified which is a member of the serine protease inhibitor family.

Methods: Vaspin cDNA was isolated by from visceral white adipose tissues of Otsuka Long-Evans Tokushima Fatty (OLETF) rat, an animal model of abdominal obesity with type 2 diabetes.

Results: Rat, mouse, and human vaspins are made up of 392, 394, and 395 amino acids, respectively; exhibit approximately $40 \%$ homology with $\alpha_{1}$-antitrypsin, and are related to serine protease inhibitor family. Vaspin was barely detectable in rats at 6 weeks and was highly expressed in adipocytes of visceral white adipose tissues at 30 weeks, the age when obesity, body weight, and insulin levels peak in OLETF rats. The tissue expression of vaspin and its serum levels decrease with worsening of diabetes and body weight loss at 50 weeks. The expression and serum levels were normalized with the treatment of insulin or insulin-sensitizing agent, pioglitazone, in OLETF rats. Administration of vaspin to obese CRL:CD-1 (ICR) (ICR) mice fed with high-fat high-sucrose chow improved glucose tolerance and insulin sensitivity reflected by normalized serum glucose levels. It also led to the reversal of altered expression of genes relevant to insulin resistance, e.g., leptin, resistin, TNF- $\alpha$, glucose transporter- 4 , and adiponectin. In DNA chip analyses, vaspin treatment resulted in the reversal of expression in approximately $50 \%$ of the high-fat high-sucrose-induced genes in white adipose tissues.

Conclusion: These findings indicate that vaspin exerts an insulin-sensitizing effect targeted towards white adipose tissues in states of obesity.

Vaspin belongs to the 'serpin' super-family. Serpins are known to act as suicidal inhibitors but the inhibitory activity of vaspin remains unknown. It could act as counteracting aberrations seen in the metabolic syndrome by sensitizing insulin action. It comes from the visceral adipose tissue and would act primarily on the white adipose tissue, but the detailed physiology of this new hormone remains to be described not only in rodents but also in humans.

The relevance of the hormone remains to be proven but the concept is stimulating because genetic variants of very few molecules acting as inhibitors have been implicated so far in the etiology of insulin resistance. The best example is ENPP-1 [19], an insulin receptor inhibitor in which polymorphism has been identified in childhood obesity and type 2 diabetes and which is reported in Barbara Heude and Ken Ong's chapter 'Population Genetics and Pharmacogenetics' [pp. 117-129]. 


\section{References}

1. Druet C, Dabbas M, Baltakse V, Payen C, Jouret B, Baud C, et al: Insulin resistance and the metabolic syndrome in French obese children. Clin Endocrinol 2006, in press.

2. Caprio S, Bronson M, Sherwin RS, Rife F, Tamborlane WV: Co-existence of severe insulin resistance and hyperinsulinaemia in pre-adolescent obese children. Diabetologia 1996;39:1489-1497.

3. Caprio S, Hyman LD, Limb C, McCarthy S, Lange R, Sherwin RS, et al: Central adiposity and its metabolic correlates in obese adolescent girls. Am J Physiol 1995;269:E118-E126.

4. Cruz ML, Weigensberg MJ, Huang TT, Ball G, Shaibi GQ, Goran MI: The metabolic syndrome in overweight Hispanic youth and the role of insulin sensitivity. J Clin Endocrinol Metabol 2004;89:108-113.

5. Gungor N, Bacha F, Saad R, Janosky J, Arslanian S: Youth type 2 diabetes: insulin resistance, $\beta$-cell failure, or both? Diabetes Care 2005;28:638-644.

6. Weiss R, Dufour S, Taksali SE, Tamborlane WV, Petersen KF, Bonadonna RC, et al: Prediabetes in obese youth: a syndrome of impaired glucose tolerance, severe insulin resistance, and altered myocellular and abdominal fat partitioning. Lancet 2003;362:951-957.

7. Balkau B, Eschwege E: The metabolic syndrome. Lancet 2005;366:1921; author's reply 1923-1924.

8. Gale EA: The myth of the metabolic syndrome. Diabetologia 2005;48:1679-1683.

9. Grundy SM: Metabolic syndrome scientific statement by the American Heart Association and the National Heart, Lung, and Blood Institute. Arterioscler Thromb Vasc Biol 2005;25:2243-2244.

10. Reaven GM: The metabolic syndrome: requiescat in pace. Clin Chem 2005;51:931-938.

11. Reynolds K, Muntner P, Fonseca V: Metabolic syndrome: underrated or underdiagnosed? Diabetes Care 2005;28: 1831-1832.

12. Ravelli ACJ, van der Meulen JHP, Michels RP, Osmond C, Barker DJP, Hales CN, et al: Glucose tolerance in adults after prenatal exposure to famine. Lancet 1998;351:173-177.

13. Soto N, Bazaes RA, Pena V, Salazar T, Avila A, Iniguez G, et al: Insulin sensitivity and secretion are related to catch-up growth in small-for-gestational-age infants at age 1 year: results from a prospective cohort. J Clin Endocrinol Metabol 2003;88:3645-3650.

14. Pettitt DJ, Aleck KA, Baird HR, Carraher MJ, Bennett PH, Knowler WC: Congenital susceptibility to NIDDM. Role of intrauterine environment. Diabetes 1988;37:622-628.

15. Pettitt DJ, Knowler WC: Long-term effects of the intrauterine environment, birth weight, and breast-feeding in Pima Indians. Diabetes Care 1998;21(suppl 2):B138-B141.

16. Randle PJ, Newsholme EA, Garland PB: Regulation of glucose uptake by muscle. 8. Effects of fatty acids, ketone bodies and pyruvate, and of alloxan-diabetes and starvation, on the uptake and metabolic fate of glucose in rat heart and diaphragm muscles. Biochem J 1964;93:652-665.

17. Ek J, Andersen G, Urhammer SA, Hansen L, Carstensen B, Borch-Johnsen K, et al: Studies of the Pro12Ala polymorphism of the peroxisome proliferator-activated receptor- $\gamma_{2}$ gene in relation to insulin sensitivity among glucose tolerant Caucasians. Diabetologia 2001;44:1170-1176.

18. Lindi VI, Uusitupa MI, Lindstrom J, Louheranta A, Eriksson JG, Valle TT, et al: Association of the Pro12Ala polymorphism in the PPAR $-\gamma_{2}$ gene with 3-year incidence of type 2 diabetes and body weight change in the Finnish Diabetes Prevention Study. Diabetes 2002;51:2581-2586.

19. Meyre D, Bouatia-Naji N, Tounian A, Samson C, Lecoeur C, Vatin V, et al: Variants of ENPP1 are associated with childhood and adult obesity and increase the risk of glucose intolerance and type 2 diabetes. Nat Genet 2005;37:863-867. 
